\title{
Response input interface
}

\author{
RONALD V. FALCONE, DONALD A. WARREN, and ROBERT A. ROSELLINI \\ State University of New York, Albany, New York
}

A circuit for interfacing experimental response signals with logic apparatus that controls experimental events is described. The unit shapes and holds the signal until its presence has been detected by the controlling program.

One of the most basic functions that must be implemented in the psychological laboratory is the interfacing of response signals from an experimental environment with the logic apparatus that controls the experimental events. Historically, this function has been implemented by pulse-forming circuitry. When a microcomputer is used for on-line control of experiments, it is often essential that the response interfacing unit be capable not only of shaping the signal, but also of holding it until its presence has been detected by the controlling program. Such a reset function is essential if the microcomputer 1/O-port interface does not have full handshaking capability. The present article describes a response interface circuit that can be constructed easily in any electronic shop.

\section{COMPONENT FUNCTIONS}

Three discrete pieces of circuitry are coordinated to interface a response signal with a computer port: (1) a Schmitt trigger, (2) a one-shot timer, and (3) a flip-flop.

The Schmitt trigger is used to eliminate switch bounce, which occurs whenever a mechanical contact is opened or closed, since this could be registered as multiple responses by the computer program. The oneshot provides a fixed-length output for any variablelength input, thereby shaping the input signal. The flipflop is implemented using two NAND gates. The inputs are SET and RESET, and the outputs are $Q$ and NOT $Q$. Table 1 shows the relationship of the input and output states for the flip-flop. As can be seen, SET and RESET are normally high, and a ground signal is used to lock NOT $Q$ in the desired state. The inherent undesirable condition of both the inputs' being simultaneously low in flip-flop circuitry is not a problem in the present application, since the computer's response time is far faster than the possible occurrence of the response.

\section{CIRCUIT DESCRIPTION}

The discrete functions discussed above are available on integrated circuit chips. We use a 74 LS132 for the

The authors' mailing address is: Department of Psychology, State University of New York, Albany, NY 12222.
Table 1

Truth Table for the Flip-Flop Circuit

\begin{tabular}{ccccc}
\hline \multicolumn{2}{c}{ Input } & & \multicolumn{2}{c}{ Output } \\
\cline { 5 - 5 } SET & RESET & & Q & NOT Q \\
\hline High & High & & \multicolumn{2}{c}{ No change } \\
High & Low & & Low & High \\
Low & High & & High & Low \\
Low & Low & & Indeterminate \\
\hline
\end{tabular}

Schmitt trigger, which has four triggers per chip with inverted outputs. These outputs must be reinverted to satisfy the input requirement of the one-shot (74LS123), which has dual circuits. The 7400 chip, which is a quad NAND gate, is used for the flip-flops and Schmitttrigger output inverters.

Figure 1 shows the logic and circuit diagram of the interface. The length of the output of the one-shot is controlled by a resistor-capacitor network comprising a 1 -kohm resistor and a 33-microF capacitor. This can be varied to suit particular needs by changing the resistorcapacitor network [i.e., time $=($ resistor $\mathrm{x}$ capacitor $) / 2$ ] . The output pulse is sent to the flip-flop, which is isolated from external voltages by diodes. We use the $1 \mathrm{~N} 4005$, but any of the $1 \mathrm{~N} 4000$ series should be adequate. The current is controlled by a $220-0 h m$ resistor and is split between the LED (used for visual verification of a response) and the remainder of the circuit. The voltage supply for this circuit must be $+5 \mathrm{~V} \mathrm{dc}$ with a ground common to the rest of the system, including the response manipulandum and the computer $\mathrm{I} / \mathrm{O}$ interface port.

For most efficient use of the gates, a set of four, or multiple of four, circuits can be combined on one board. This is ideal for 8-bit microcomputers, since they are capable of accepting 8 simultaneous bits of input from the port device. The physical layout of such a circuit is shown in Figure 2.

\section{CIRCUIT OPERATION}

The sequence of events associated with the response interface starts with the controlling program's sending a ground pulse to the RESET terminal. This sets the out- 

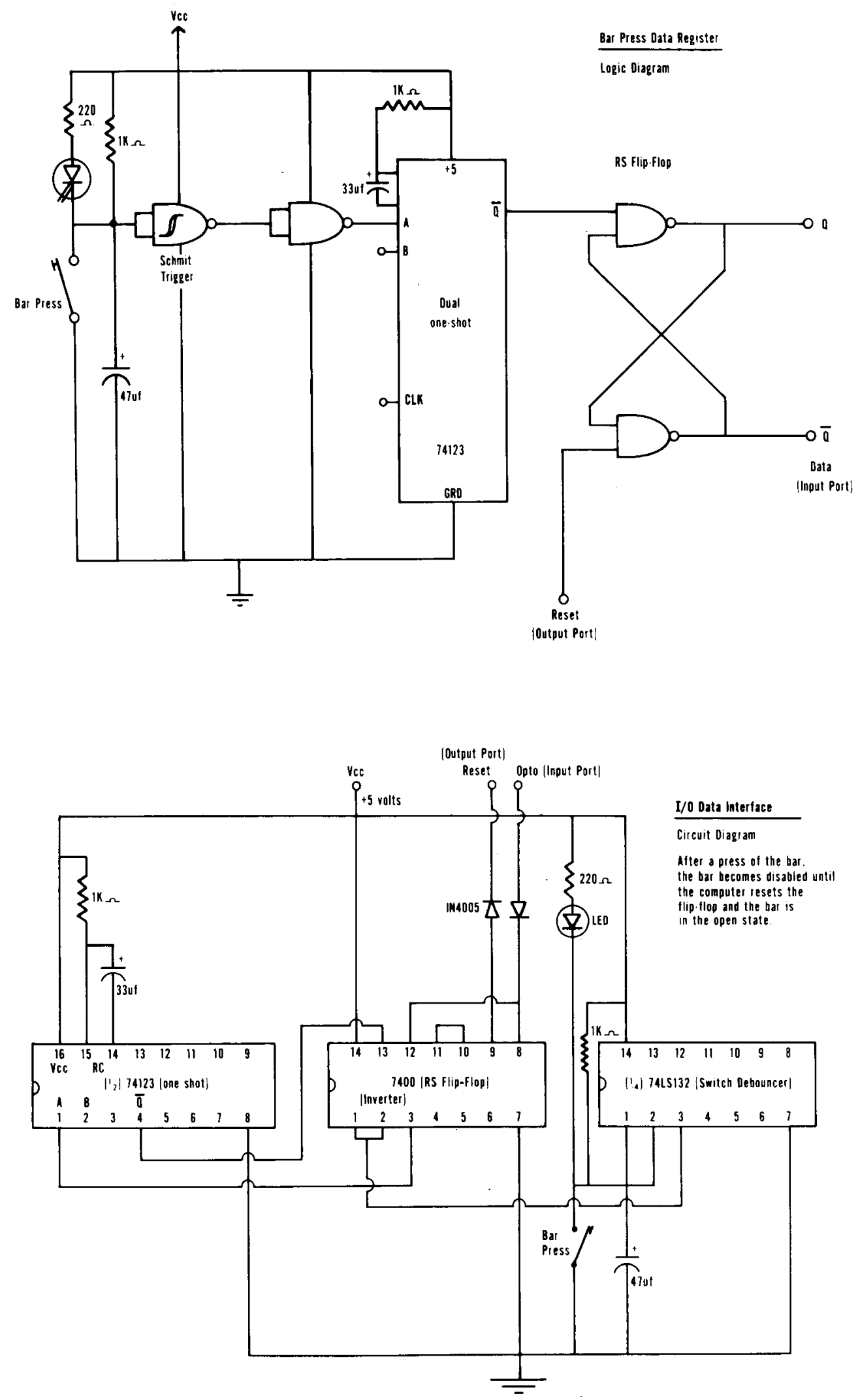

Figure 1. Logic (top panel) and wiring (bottom panel) diagram of one circuit of the response interface. 


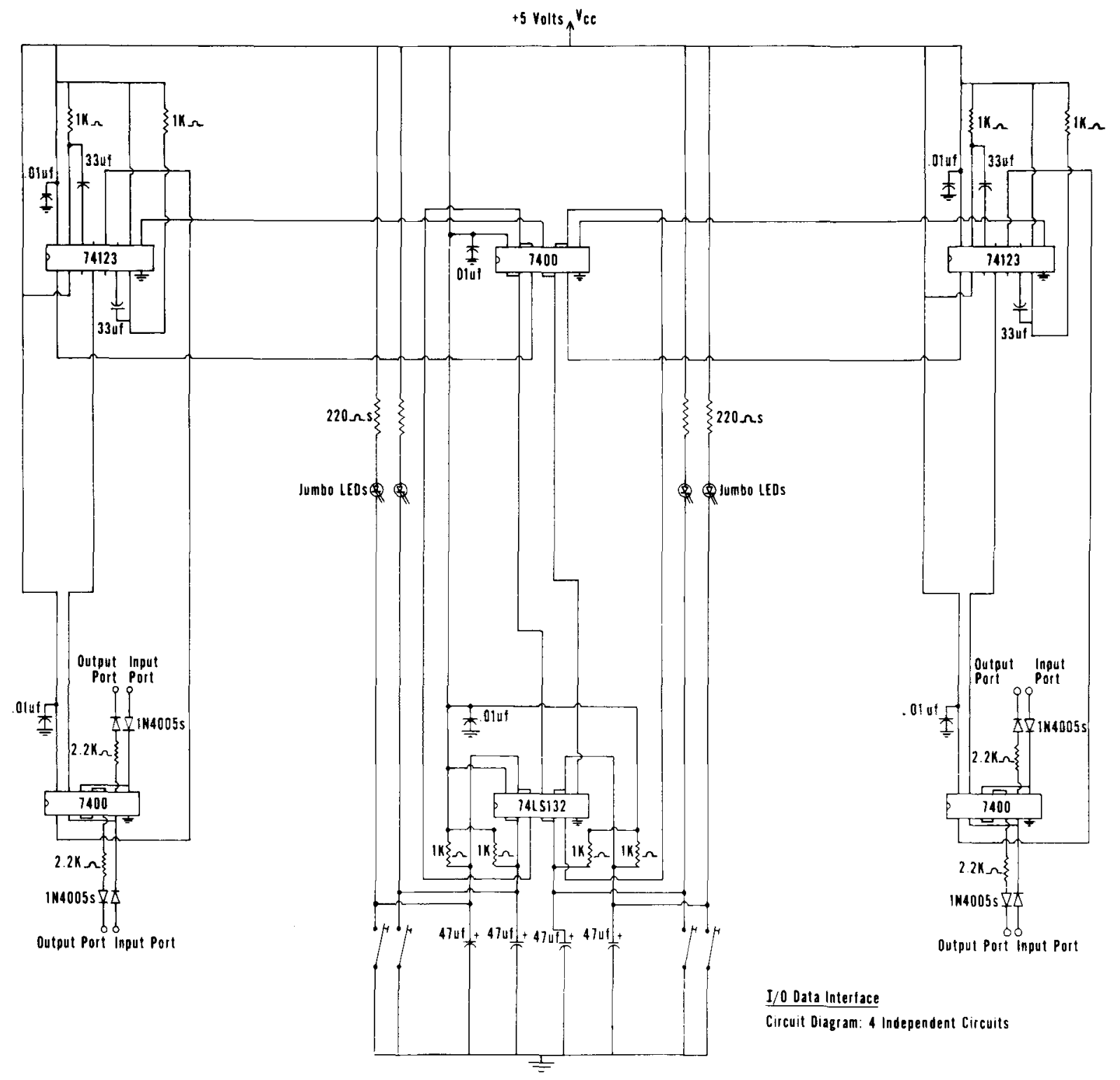

Figure 2. Wiring diagram for a response interface unit consisting of four independent circuits.

put of the interface to the "high" state. The occurrence of a response in the experimental environment introduces a ground (low signal) to the input terminal of the interface, which responds with a low signal at the output. This low condition is the signal to the logic controlling device that a response has occurred. Upon recognition of the response, the controlling device should send a low signal to the reset terminal of the interface. This will reinitiate the cycle by setting the output of the interface to the high state and readying it for the next response.

(Manuscript received March 4, 1983; revision accepted for publication April 13, 1984.) 